\title{
Increasing Mobility of Tracked Vehicles during Curvilinear Motion by Redistributing Power Flows
}

\author{
I.A. Taratorkin ${ }^{1}, M . V$. Vyaznikov $^{1}$, and A.M. Vyaznikov ${ }^{1, *}$ \\ ${ }^{1}$ Institute of Engineering Science UB RAS, Yekaterinburg
}

\begin{abstract}
A kinematic diagram of an electromechanical transmission of a high-speed tracked vehicle with two traction electric motors, on-board gearboxes and a ZK-type differential turning mechanism, which makes it possible to effectively distribute power flows between the sides during curvilinear motion, is proposed.
\end{abstract}

\section{Introduction}

At present, the possibilities of increasing average speeds of high-speed tracked vehicles when turning are limited by many factors, including the design of the turning mechanism, control system, and peculiarities of tracks and ground interaction. When turning at speeds above 35$40 \mathrm{~km} / \mathrm{h}$, the driver cannot take proactive actions to prevent sideslip (skidding) due to the delay in the driver's own reaction to a change in the movement trajectory, as well as due to the delay in the reaction of the vehicle to a change in the position of the control (steering wheel). The main limitation of the speed of movement is partial sideslip, which is characterized by the loss of traction of the tracks with the ground and the beginning of uncontrolled sideslip under certain parameters of the vehicle turning in specific road and ground conditions. At the same time, the results of studies of the steering dynamics accumulated to date show the dependence of stability of movement on displacement of the turning pole and the sideslip of the lower track wheels.

In general, the problems of controllability of high-speed tracked vehicles were considered by E.E. Alexandrov, A.A. Blagonravov, S.E. Burtsev, V.V. Guskov, V.B. Derzhanskiy, N.A. Zabavnikov, A.M. Kaufman, V.I. Krasnenkov, F.A. Opeiko and other prominent scientists in the field of transport engineering. The main directions of research were the choice of the optimal kinematic scheme of the steering device, which provides not only the necessary parameters of curvilinear motion, but also minimizes the required turning power.

There is a classification of the steering devices (SD) of tracked vehicles according to a number of characteristics. In particular, according to the nature of the change in the gravity center speed of the vehicle when turning the SD, so it can be divided into the following groups:

\footnotetext{
* Corresponding author: priss1912@yandex.ru
} 
- devices that keep the gravity center speed of the vehicle unchanged when turning, which include all differential steering mechanisms;

- devices that preserve the speed of the running tracks when turning, which include all independent SD of various types, including planetary SD, side clutches, and onboard transmissions.

By the nature of the control of the driving wheel rotation speed, the following groups of SD are distinguished:

- differential, which provide a symmetric decrease in the frequency of rotation of the leading wheel of the trailing side and increase the frequency of rotation of the driving wheel of the leading side. In this case, the theoretical speed of the gravity center of the vehicle when turning remains unchanged;

- independent (side), providing turning, as a rule, by reducing the speed of the leading wheel of the trailing side while maintaining the same speed of the driving wheel of the leading side. As a result, the theoretical speed of the vehicle's center of gravity when turning is reduced. Such mechanisms include all independent SD of various types, including planetary $\mathrm{SD}$, side clutches, and onboard transmissions.

The need to increase the mobility of tracked vehicles requires solving a set of problems, and among them, the most important is the choice of a steering device, taking into account the type of power plant and transmission.

The onboard (independent) steering device is used on all-terrain vehicles and snow and swamp vehicles intended for operation in the northern hard-to-reach areas, as well as municipal and firefighting equipment on a tracked platform. Such device has a simpler design and ensures that during the turn, the leading side of the vehicle maintains the forward speed equal to the speed of the straight-line motion of the vehicle before entering the turn, while automatically reducing the speed of the geometric center of the vehicle.

\section{Main part. Substantiation of the kinematic diagram of the steering device}

In the design of high-speed tracked vehicles, transmissions, including a full-flow complex lockable hydrodynamic transmission, a central, usually planetary gearbox and a two-flow stepless steering device, consisting of a hydrostatic transmission with a variable pump and an unregulated motor having a mechanical differential connection with two summing planetary mechanisms, have become widespread. When using the differential steering device, the speed of the trailing side decreases and the same magnitude increase is noted in the speed of the leading side at a constant speed of the center of the vehicle (Fig. 1). This takes place, without taking into account slipping of the tracks, under the condition of constant high-speed operation of the engine, constant gear ratio of the full-flow hydrodynamic transmission and absence of shifting in the gearbox when there is a change from the straight-line motion of the tracked vehicle into the turn. At the same time, a relatively high level of the required installed power of the hydrostatic transmission is due to the need for it to work simultaneously both to brake the trailing side and to accelerate the leading side. 


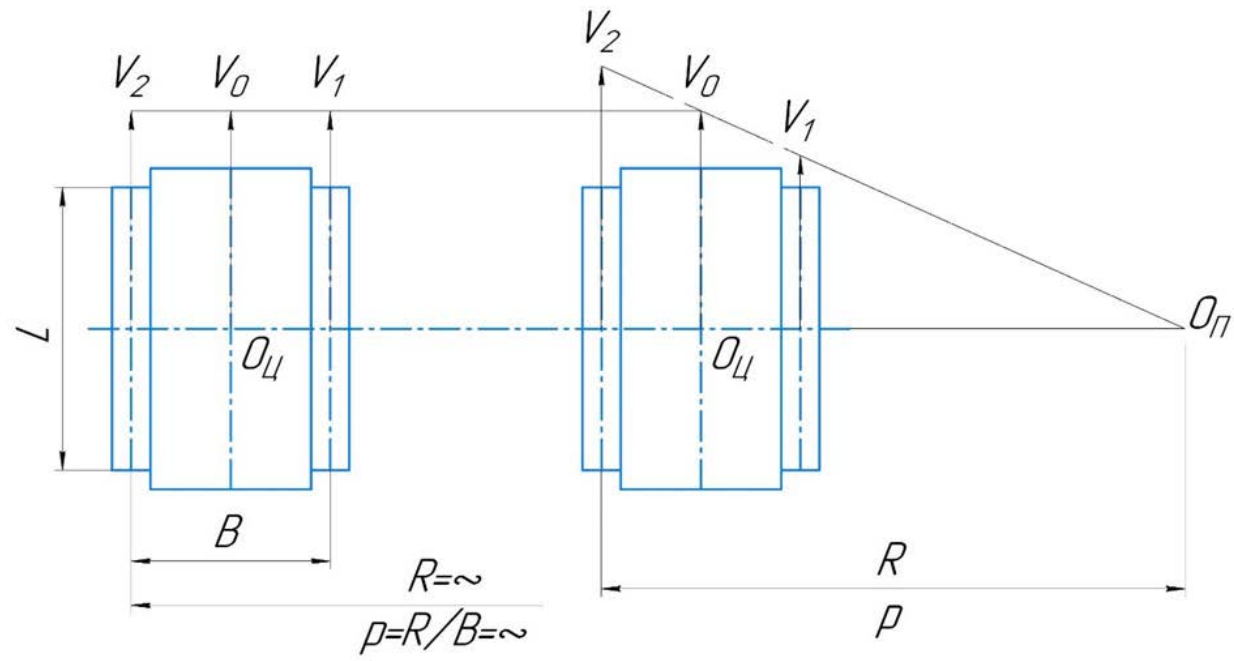

Fig. 1. Differential way of steering the tracked vehicle

$\mathrm{V} 2$ is the speed of the running track; V1 is the speed of the trailing track; V0 is the speed of the center of mass; Оц - center of mass; Оп - center of turn; В - track width; L - track length

The side or independent way of turning ensures that in a turn the speed of the forward motion is maintained by the leading side of the vehicle, equal to the speed of the straight-line motion of the vehicle before entering the turn, while the speed of the geometric center of the vehicle is automatically reduced. When turning with a radius equal to the car's track, the speed of the geometric center of the vehicle will be half that of a vehicle with a differential steering device.

The plan of speeds of the forward motion of the geometric center and the sides of the vehicle with an onboard (independent) SD is shown in Fig. 2.

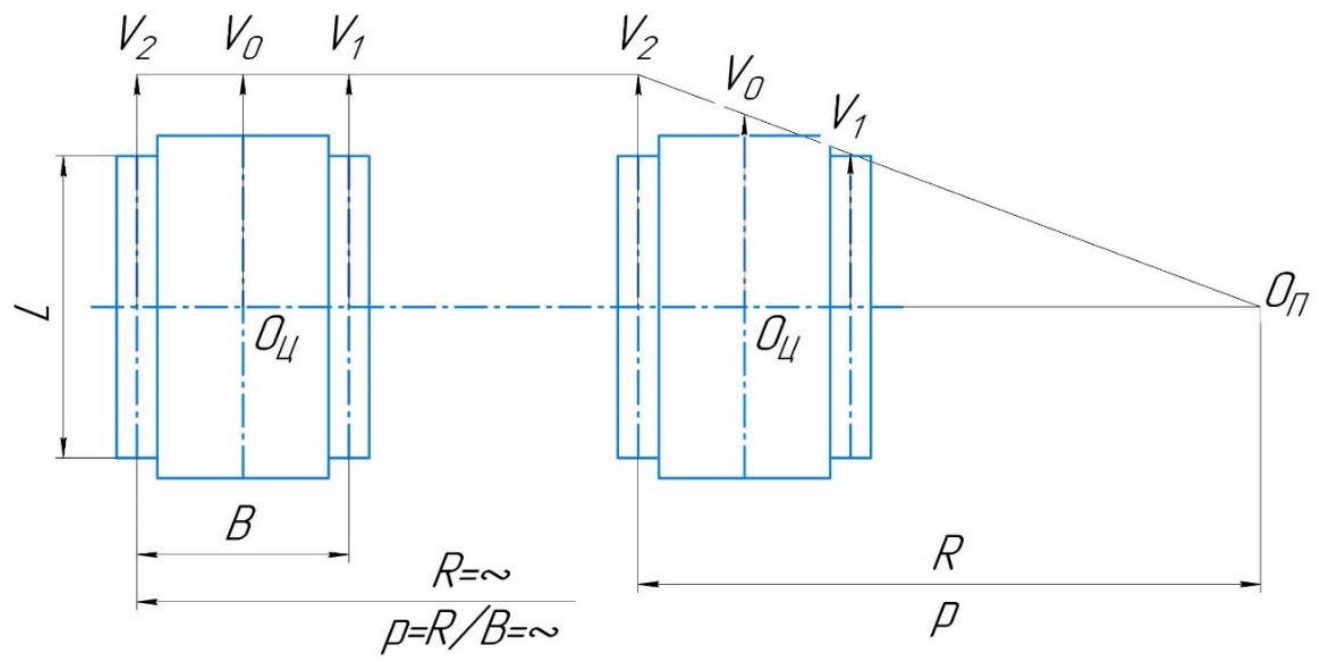

Fig. 2. Onboard (independent) way of steering the tracked vehicle 
The well-known advantage of the differential turning method, which consists in the possibility of movement of the vehicle in a turn at a higher speed than with other known methods - onboard or independent, cannot always be realized, since the limiting speeds of the vehicle's turning are not limited by the way of turning, but by the possibility of maintaining stability of movement and controllability of the vehicle in a turn [1], [2], [3]. Another problem is to provide the possibility of a stepless change in the turning radius in combination with a high speed of response in transient processes [4], which can be solved by using a combined multi-stream steering device with one-way kinematic connections [5].

When turning the vehicle at high speeds, with small turning radii, on slippery and weakbearing soils, in order to eliminate the danger of the vehicle falling off into an uncontrollable side skid, it is necessary to reduce the speed of the vehicle in advance, before entering the turn, switch the gearbox to lower gears, i.e., turning at much slower speeds than the differential steering alone can provide. Moreover, with the side turning method, the motion speed of the vehicle's center in the turn is automatically reduced with a decrease in the turning radius.

Currently, one of the ways to increase the mobility of high-speed tracked vehicles is the use of a hybrid power plant with an electromechanical transmission. This is facilitated by significant progress in the production of small-sized electric machines with high power density, electrical and electronic devices that convert electric current and control electric machines, as well as energy storage units (ESU) with high energy intensity, allowing a significant number of recharges. Conversion of mechanical energy into electrical energy provides infinitely variable torque and speed control of the driving wheels in a wide range. In addition, there are ample opportunities for using the spaced-apart arrangement of units and systems of the engine-transmission unit, which makes it possible to free up additional internal volume in the vehicle's body to accommodate the payload. An important issue is the reasonable choice of the power of the traction electric drive. With the classic on-board (independent) two-machine electromechanical transmission scheme, one to ensure the turning of the vehicle for the entire set of road and ground conditions, the maximum power of each traction motor must be at least $70 \%$ of the generator power. To reduce the weight and size characteristics of electrical machines, it is proposed to combine them with a differential mechanical link based on a ZK-type steering device. In this case, the required power of each traction motor must be $50 \%$ of the generator power.

A kinematic diagram of the electromechanical transmission includes two traction electric motors, two 2-stage onboard transmissions and a ZK-type steering device (Fig. 3). With equal rotation speeds of the traction motors transmitting rotation to the sun gears of the differential, the linear motion of the tracked vehicle is ensured. With a difference in the rotational speeds of the traction motors, it is necessary to turn the vehicle with the required radius, a ZK-type steering device provides recuperation of power flows between the sides, providing the required turning moment. 


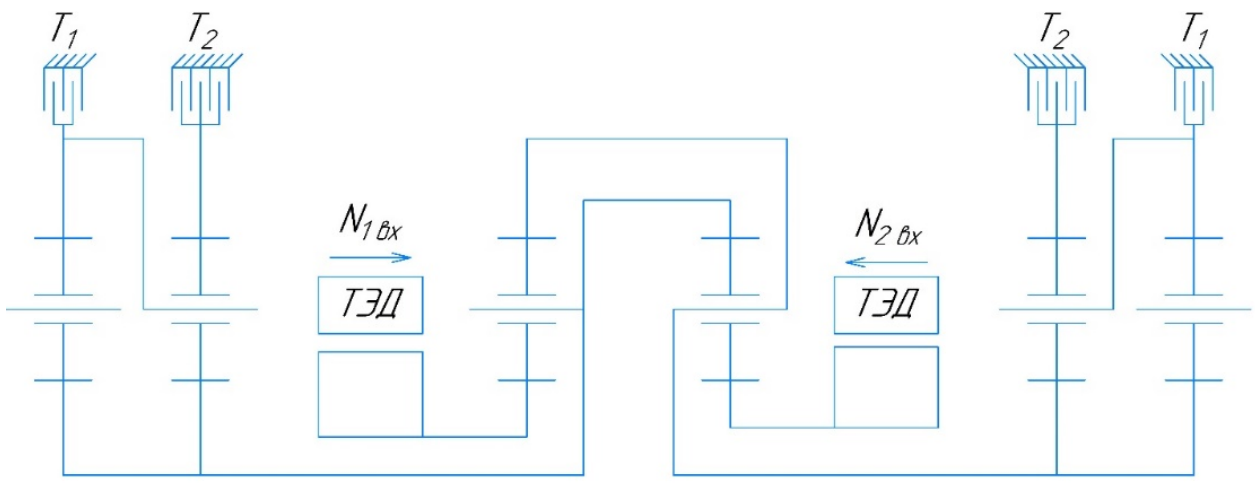

Fig. 3. Kinematic diagram of ЕМВ: ТЭД - traction motor; Т1 - brake for switching on the 1st range; $\mathrm{T} 2$ - brake for switching on the 2nd range; N1 and N2 - power flows from traction motors.

To improve the quality of transient processes when controlling the vehicle in a turn, it is proposed to introduce a booster circuit to increase sensitivity of the system to the control action set by the driver through the turning control body or generated by the microcontroller when implementing the control algorithm for the robotic tracked platform [6], [7].

\section{Conclusions}

The general concept of creating a tracked vehicle equipped with 2 electric motors and a ZKtype planetary power recovery mechanism between the sides has been worked out. Although in many countries there have been attempts to manufacture tracked vehicles with an electromechanical transmission, at present, the traction electric drive for tracked vehicles is widely used only on light, low-speed mobile robots. It still has not found wide distribution due to a number of shortcomings:

- large weight and dimensions;

- complexity of operation;

- lack of acceptable energy storage units, low autonomy;

- low efficiency due to multiple energy conversion;

- inconsistency of characteristics of electric motors of most types with the changing conditions of motion of tracked vehicles;

- need to use auxiliary devices (in particular, expensive and large-sized electrical converters).

It should be noted that some of these shortcomings have now been practically eliminated. Thus, the use of semiconductor power devices with microprocessor control made it possible to obtain mechanical characteristics for electric motors of many types, which increase the efficiency of their use on tracked vehicles.

It is also important that in order to implement this concept, it is necessary to solve a whole range of technical and layout problems. However, given the significant improvement in technologies for the production of generators, electric motors and batteries over the past decades, its implementation becomes possible.

In addition to improving the transmission of tracked vehicles directly, there is also the prospect of improving the motion control system by reading the motion parameters in real time with a parallel adjustment of the control action. This technology will automate the complex operation of all units and adjust the control action of the driver in order to increase mobility of tracked vehicles. 


\section{References}

1. Opeyko F.A. Wheeled and tracked. - Minsk: AN BSSR, 1960.

2. Krasnenkov V.I. Fundamentals of the theory of controllability of transport tracked vehicles. - M.: N.E. Bauman MSTU, 1977 . - 82 p.

3. Blagonravov A.A., Derzhanskiy V.B. Dynamics of the controlled movement of the tracked vehicle. - Kurgan: Publishing house of the Kurgan Machine-Building Institute, 1995. - 162 p.

4. Vyaznikov M.V. Experimental studies of dynamics of the curvilinear movement of tracked vehicles // Selected works of the Russian school 'To the 70th anniversary of G.P. Vyatkin'. - M: RAS Publ., 2005, pp. 501-509.

5. Vyaznikov M.V. Improvement of steering control systems for multi-purpose tracked vehicles // Collection of scientific works of MADI (GTU). - M.: MADI, 2008, pp. 144153.

6. Vyaznikov M.V. Perspective control systems for movement of tracked vehicles // Vestnik mashinostroeniya [Bulletin of mechanical engineering], No. 12, 2004, pp. 26-29.

7. Vyaznikov M.V. Problems of synthesis of intelligent control systems for tracked vehicles / Automotive industry, 2005, No. 6. 